\section{IMMUNE STATUS TO MEASLES, RUBELLA, AND VARICELLA-ZOSTER AMONG NEWLY RECRUITED HEALTHCARE WORKERS AT KING ABDULAZIZ MEDICAL CITY, JEDDAH, SAUDI ARABIA}

Mohammed AbdalAziz, Fayssal Farahat, John Ossenkopp, Majid AlThaqafy, Omaima Hassan, Asim AlSaedi. Infection Prevention and Control King Abdulaziz Medical City, Jeddah

\subsection{6/bmjoq-2019-PSF.31}

Background Measles, rubella, and varicella-zoster are vaccine-preventable diseases that have the potential of transmission in healthcare settings. Healthcare professionals are at high risk of exposure to these viral diseases and should be immunized. Screening and vaccination of newly recruited healthcare workers (HCWs) is considered a cost effective intervention. All newly recruited $\mathrm{HCW}$ are required to have physical and serological assessment before they start working. Serology screening covers several vaccine-preventable diseases including measles, rubella, and varicella. This study aimed to identify the immune status to these diseases among the newly recruited $\mathrm{HCW}$ at King Abdulaziz Medical City, Jeddah (KAMC-J).

Methods A retrospective review of the employees' medical records was conducted. Data were extracted from the electronic medical records and database of pre-employment assessment. Data were collected on demographic data (age, sex, and nationality) and IgG antibodies of measles, rubella, and varicella-zoster virus.

Results During the study period, 673 newly recruited HCWs were screened. The mean age was 26.5 (SD 5.5) years, and two-thirds $(63 \%)$ of the participants were female. The majority of participants were immune against measles (87.3\%), rubella (87.8\%), and varicella (93.2\%). Immunity (positive $\mathrm{IgG}$ ) against measles, rubella, and varicella was higher in females than in males. Similarly, measles and rubella IgG was higher in older participants (older than 25 years of age); however, varicella IgG was higher among younger participants (25 years old or younger). Measles-positive IgG ranged from $78.9 \%$ to $96.9 \%$; the lowest was among non-clinical HCWs and the highest was among nurses. Rubella-positive IgG ranged from $85.7 \%$ to $93.8 \%$; the lowest was among allied health staff and the highest was among nurses. Varicella-positive IgG ranged from $91.2 \%$ to $95.5 \%$, the lowest was among allied health staff and the highest was among physicians. Among the new Saudi employees, prevalence rates of IgG for measles, rubella, and varicella were $85.1 \%, 86.7 \%$, and $93.1 \%$, respectively, which were lower than those for nonSaudi HCWs.

Conclusion This study revealed good immunity coverage for measles, rubella, and varicella among newly recruited HCWs; however, the allied health staff and non-clinical HCWs should receive more attention to optimize their vaccination status. Immunization of new HCWs should be enhanced as an essential component of workers' protection to reduce the risk of transmission and burden on the healthcare system.

\section{HEPATITIS B SEROLOGICAL STATUS AMONG A POPULATION SCREENED IN THE MINISTRY OF NATIONAL GUARD HEALTH AFFAIRS IN SAUDI ARABIA BETWEEN 2016 AND 2018}

John Ossenkopp, Fayssal Farahat, Mohammed Abdel Alaziz, Majid AlThaqafy, Asim AlSaedi. Infection Prevention and Control King Abdulaziz Medical City, Jeddah

\subsection{6/bmjoq-2019-PSF.32}

Background Hepatitis B viral infection represents a major public health problem worldwide. Despite being considered a vaccine- preventable disease; prevalence is still high in many regions. Most people are unaware that they are infected, and are frequently diagnosed during routine screening or as a work-up of advanced hepatic disease. The current study aimed to evaluate the proportion of the population currently infected, with past infection, or noninfected, and to determine the proportion of the population that is immunized.

Methods A retrospective descriptive study was done, reviewing the serological markers for hepatitis $\mathrm{B}$ requested among the population who attended the MNGHA, Jeddah. Data from people screened between 2016 and 2018 were included in this study. Serological markers for hepatitis B included hepatitis B surface antigen (HBsAg), hepatitis B core antibodies (HBcAbs), and hepatitis B surface antibodies (HBsAbs).

Results Among 8370 people screened for hepatitis B, 312 $(3.72 \%)$ individuals were found to be infected with hepatitis $\mathrm{B}$ ( $\mathrm{HBsAg} / \mathrm{HBcAb}$ positive, or at least $\mathrm{HBsAg}$ positive). The majority $(92.34 \%, \mathrm{n}=7729)$ was not infected (HBsAg negative/HBcAb negative, or $\mathrm{HBsAg}$ negative/ $\mathrm{HBcAb}$ positive/HBsAb positive as past infection, and $\mathrm{HBcAb}$ negative/ $\mathrm{HBsAb}$ positive as immune). Serological status of infection of the remaining $3.93 \%$ was unknown (only $\mathrm{HBsAb}$ negative tested, or isolated core: $\mathrm{HBcAb}$ positive/HBsAg negative/HBsAb negative [possible occult infection], or only $\mathrm{HBcAb}$ positive tested). Among subjects screened for HBsAbs ( $\mathrm{n}=5619), 3382$ (60.19\%) were immune (HBsAb $>10 \mathrm{IU} / \mathrm{mL}$ ). Of 4960 individuals tested for HBcAbs, 862 $(17.38 \%)$ were found to be positive, exposed to hepatitis $\mathrm{B}$ virus (active, occult, or past infection). The majority of infected individuals were Saudi (94.6\%) and 57.6\% were male; however, $75 \%$ of the population screened were Saudi, with $4.66 \%$ of infection among Saudis. Regarding the population exposed to hepatitis B (HBcAbs positive tested), 22.28\% were Saudis, 20.08\% from the Philippines, $16.90 \%$ from Pakistan, $14.52 \%$ from Egypt, and $9.55 \%$ from India, from the most frequently exposed nationalities. Among immune people, $54.3 \%$ were Saudi and $58.5 \%$ were female.

Conclusion Despite the available vaccination for hepatitis $B$ virus, more than $3 \%$ of the population screened was infected, and more than $17 \%$ had been exposed to the virus (HBcAbs positive). Screening for hepatitis $\mathrm{B}$ virus, early identification of infected individuals, and vaccination for the non-immune population must be encouraged. 